\title{
Kinematic Analysis of Take off Technique on the Performance of the Fosbury Flop
}

\author{
Dr.C.VenkateswaraRao ${ }^{1}$, Dr.Ch. Raja rao $^{2}$ \\ ${ }^{1}$ Asst.Prof.G.S.DepartmentJubail Industrial College,KSA \\ ${ }^{2}$ Lecturer in Physical Education, GDC,Mahabubabad,Wgl,Telangana,India.
}

\begin{abstract}
The high jump is one of the most technical and complex event of the athletics. Top high jumper's in the world use one of the versions of Fosbury Flop technique, this proved the most efficient so far. This technique comprises the preparation for the touchdown, take off, the flight phase, bar clearance and landing. It is not easy to learn and perform, since it requires some special skills. Its mechanical and dynamic analysis reveals movements of an exceptional difficulty that cannot be so easily assimilated by most jumpers. Certain "time" of execution (particularly those made over the bar) requires a psycho motor control and an extreme sensibility that can't be easily reached by everyone. Thirteen male national high jumpers were selected for this study and get videotaped with three high speed video cameras during their competitive performance in the men's high jump final during the 52nd National open athletics championship in outdoor with the informed consent of the athletes. In this research we used three Panasonic-AG-DVX-102B, F11 sensitivity, high image quality, camcorders, Quintic Biomechanics 21 motion analysis software.
\end{abstract}

\section{Introduction}

To clear a high jump bar, it is necessary to drive the center of mass (COM) of the athlete as high as possible. It is also necessary to move the body in the air in a way that will allow the athlete to clear a bar set as close as possible to the peak height reached by the COM. For a given peak height reached by the COM lowering some parts of the body allows other parts to go higher. This was the mechanical principle that has improved the effectiveness of the bar clearance over the years.

If a high jumper remains in a straight vertical position after taking off from the ground, the height of the bar that the feet will be able to clear will be far below the peak height of the COM. The simplest improvement over this was a technique in which the athlete flexes at the hips and knees to adopt a squatting position at the peak of the jump. We could call this the "legs-up" technique. Since the peak height of the COM can't be changed after the athlete leaves the ground, the lifting of the legs is accompanied by a lowering of the trunk. This technique greatly increases the height of the bar that has to be cleared.

The major purpose of this study was to investigate the take-off motion at the end of the last stride and identify the characteristics for optimum performance executed by Fosbury-flop jumpers, using 2-dimensional videography. The best jumps of thirteen male high jumpers were filmed at an official track meet by two highspeed cameras. The results suggested that to lower the center of gravity during the touchdown, Fosbury flop jumpers' takeoff leg was planted ahead of the torso by leaning the body backward.

\section{Selection of Subjects:}

\section{Methodology}

Thirteen male national high jumpers were selected for this study and get videotaped with three high speed video cameras during their competitive performance in the men's high jump final during the 52nd National open athletics championship in outdoor with the informed consent of the athletes.

\section{Tools and equipment's:}

The experimental apparatus used in this research work were three Panasonic-AG-DVX-102B, F11 sensitivity, high image quality, camcorders, Quintic Biomechanics v21 motion analysis software.

\section{Collection of data and filming protocol:}

For quantitative video analysis certain procedures must be followed carefully, at both the video recording and digitizing stages, to minimize the systematic and random errors in the digitized co-ordinates.

For the collection of data three Panasonic camcorders were used. First camcorder was fixed at left standard line with a distance of 9.90 meters from the left upright for left foot takeoff jumpers, the second camcorder was fixed perpendicular to the bar with a distance of 15.25 meters from the bar and third camcorder mounted at right standard line with a distance of 9.90 meters from the right upright for right foot takeoff jumpers. Three camcorders captured the video clippings of Fosbury flop jumper's last stride. Each jump image 
analysis started prior to the end of the penultimate stride of the approach run and continued until the flight path of the center of mass had reached its peak. Prior to digitizing the jump sequences, 18 landmarks on the image (top of the head, neck, left shoulder, left hip, right hip, right shoulder, left elbow, left wrist, left hand, right elbow, right wrist, right hand, left knee, left ankle, left toe, right knee, right ankle, right toe) were digitized manually (minimum twelve frames).

The variables selected for this study during takeoff phase were:

\section{1) The height of the center of mass at touchdown (HTD) \\ 2) The height of the center of mass at toe off(HTO) Analysis}

The raw data were arranged separately, tabulated and subjected for the descriptive statistical analysis, followed by coefficient of correlation by using SPSS to distinguish if there any deviation. The researcher reached at the results of this empirical investigation which is presented by the respective tables and graphs.

Table 1: Physical characteristics of the thirteen subjects and their best performance

\begin{tabular}{|c|c|c|c|c|c|c|c|c|}
\hline Sl.No & Name of the Athlete & Age & Ht & Wt & Leg length & BMI & Training age & Best jump \\
\hline 1 & AmarnathOjha & 21 & 187 & 64 & 102 & 18.30 & 2 & 195 \\
\hline 2 & Arun Kumar & 22 & 183 & 66 & 98 & 19.71 & 8 & 200 \\
\hline 3 & Ashok.M & 27 & 180 & 66 & 95 & 20.37 & 8 & 200 \\
\hline 4 & Ch.Nikhil & 23 & 188 & 76 & 98 & 21.50 & 7 & 216 \\
\hline 5 & HarishankarRai & 29 & 177 & 72 & 87 & 22.98 & 13 & 216 \\
\hline 6 & Harshith .S & 18 & 189 & 62 & 99 & 17.36 & 2 & 216 \\
\hline 7 & Jagdeepsingh & 20 & 187 & 71 & 97 & 20.30 & 5 & 205 \\
\hline 8 & Jithinthomas & 22 & 175 & 61 & 95 & 19.92 & 8 & 222 \\
\hline 9 & K.Gotham & 22 & 180 & 65 & 93 & 20.06 & 5 & 205 \\
\hline 10 & K.S.R.Singh & 22 & 179 & 62 & 94 & 19.35 & 4 & 205 \\
\hline 11 & Navin.S & 23 & 180 & 73 & 92 & 22.53 & 8 & 195 \\
\hline 12 & Ritheshkumar & 22 & 180 & 62 & 90 & 19.38 & 5 & 195 \\
\hline \multirow[t]{3}{*}{13} & Shaiju.A & 26 & 185 & 70 & 95 & 20.45 & 3 & 200 \\
\hline & Mean & 22.85 & 182.31 & 66.92 & 95.00 & 20.17 & 6.00 & 205.38 \\
\hline & Sd & 2.93 & 4.50 & 4.94 & 3.98 & 1.54 & 3.08 & 9.24 \\
\hline
\end{tabular}

Age unit: Years, Height/length unit: centimeters, Weight unit: Kilo grams

The data indicates that the average age of thirteen Fosbury flop high jumpers is $22.85 \pm 2.93$ years with an average height of $182.31 \pm 4.50$; average weight is $66.92 \pm 4.94 \mathrm{~kg}$ average leg length was $95.00 \pm 3.98 \mathrm{~cm}$, average BMI is $20.17 \pm 1.54$ and training age is $6 \pm 2.93$ years. The best performance was $222 \mathrm{~cm}$.

Table No.2: touchdown variables during jumpers best performance.

\begin{tabular}{|l|l|l|l|l|l|l|l|l|}
\hline BIB No & Standing Height & Bar Height & $\%$ HTD & HVTD & VVTD & T $\left(^{\circ}\right)$ & Lean TD & TD Time \\
\hline 190 & 187 & 195 & 41 & 7.41 & 1.73 & 150.55 & 84.09 & 120 \\
\hline 728 & 183 & 200 & 38 & 7.58 & 1.5 & 146.09 & 85.14 & 80 \\
\hline 819 & 180 & 200 & 39 & 8.21 & 1.17 & 162.18 & 79.46 & 120 \\
\hline 750 & 188 & 216 & 43 & 8.04 & 2.48 & 166.87 & 82.63 & 120 \\
\hline 343 & 177 & 216 & 40 & 8.11 & 2.54 & 168.42 & 76.14 & 80 \\
\hline 591 & 189 & 216 & 43 & 7.8 & 1.54 & 150.99 & 79.11 & 120 \\
\hline 593 & 187 & 205 & 38 & 8.9 & 2.23 & 161.76 & 84.92 & 80 \\
\hline 827 & 175 & 222 & 49 & 9.13 & 2.65 & 161.8 & 77.91 & 80 \\
\hline 488 & 180 & 205 & 41 & 8.85 & 1.71 & 153.01 & 88.74 & 80 \\
\hline 345 & 179 & 205 & 41 & 7.45 & 1.93 & 151.3 & 87.24 & 160 \\
\hline 532 & 180 & 195 & 41 & 7.19 & 1.09 & 161.15 & 72.41 & 80 \\
\hline 495 & 180 & 195 & 39 & 7.48 & 1.45 & 154.08 & 79.46 & 120 \\
\hline 860 & 185 & 200 & 39 & 7.78 & 1.58 & 152.12 & 84.94 & 160 \\
\hline Mean & 182.31 & 205.38 & 40.92 & 7.99 & 1.82 & 156.95 & 81.71 & 107.69 \\
\hline SD & 4.50 & 9.24 & 2.93 & 0.63 & 0.51 & 7.04 & 4.04 & 30.04 \\
\hline
\end{tabular}

*(HTD\%) COM in relation to height of each jumper, *(HVTD) horizontal velocity at touchdown, *(VVTD) vertical velocity at touchdown, *(TD)knee angle at touchdown, *(BLTD)backward lean at touchdown, *(TD)time taken at touchdown.

Above table shows the touchdown parameters of each flopper's best jump during the competition situation. Majority of subjects maintain low COM with minimum speed loss at touchdown but knee angle at TD was not optimum level so that the negative vertical velocity was not optimum level. Majority subject's backward lean was not optimum level when compared to the elite athletes.Majority of subject's lean is not optimum at touchdown. 
Table No.3: toe off variables during jumper's best performance.

\begin{tabular}{|l|l|l|l|l|l|l|l|l|}
\hline BIB No & Standing Height & Bar Height & \% HTO & HVTO & VVTO & KATO & BLTO $\left(^{\circ}\right)$ & TO time \\
\hline 190 & 187 & 195 & 61 & 5.35 & 4.66 & 160 & 165 & 88 \\
\hline 728 & 183 & 200 & 61 & 2.11 & 5.02 & 200 & 173.29 & 86.05 \\
\hline 819 & 180 & 200 & 58 & 4.27 & 4.43 & 160 & 174.8 & 81.36 \\
\hline 750 & 188 & 216 & 68 & 5.54 & 5.42 & 160 & 178.46 & 88.62 \\
\hline 343 & 177 & 216 & 63 & 5.47 & 5.54 & 160 & 178.41 & 87.9 \\
\hline 591 & 189 & 216 & 65 & 2.39 & 3.54 & 160 & 175.6 & 87.83 \\
\hline 593 & 187 & 205 & 62 & 5.56 & 4.89 & 200 & 178.2 & 88 \\
\hline 827 & 175 & 222 & 75 & 6.13 & 6.7 & 160 & 179.23 & 89.22 \\
\hline 488 & 180 & 205 & 62 & 6.47 & 5.12 & 160 & 161.57 & 86.47 \\
\hline 345 & 179 & 205 & 65 & 3.33 & 4.63 & 240 & 176.79 & 89.26 \\
\hline 532 & 180 & 195 & 64 & 3.46 & 4.56 & 200 & 169.22 & 87.56 \\
\hline 495 & 180 & 195 & 62 & 3.52 & 4.85 & 200 & 171.87 & 81.36 \\
\hline 860 & 185 & 200 & 96 & 3.37 & 4.78 & 240 & 170.98 & 85.97 \\
\hline Mean & 182.31 & 205.38 & 66.31 & 4.38 & 4.93 & 184.62 & 173.34 & 86.58 \\
\hline SD & 4.50 & 9.24 & 2.93 & 0.63 & 0.51 & 30.72 & 4.72 & 2.61 \\
\hline
\end{tabular}

*HTD\%) COM in relation to height of each jumper, *(HVTO) horizontal velocity at toe off, *(VVTO) vertical velocity at toe off,*(TO) knee angle at toe off,*(BLTO) backward lean at toe off,*(TO) time taken at toe off.

Above table shows the toe off parameters of each flopper's best jump during the competition. Majority of subjects COM at toe off was not optimum and horizontal velocity at toe off has to reduce. It shows the majority of subjects not able to convert the horizontal velocity into vertical velocity. At toe off the knee angle and backward lean was optimum. Majority of subjects lean is not optimum at toe off.

Figure1: Height of COM at touchdown and toe off during the best jumps.

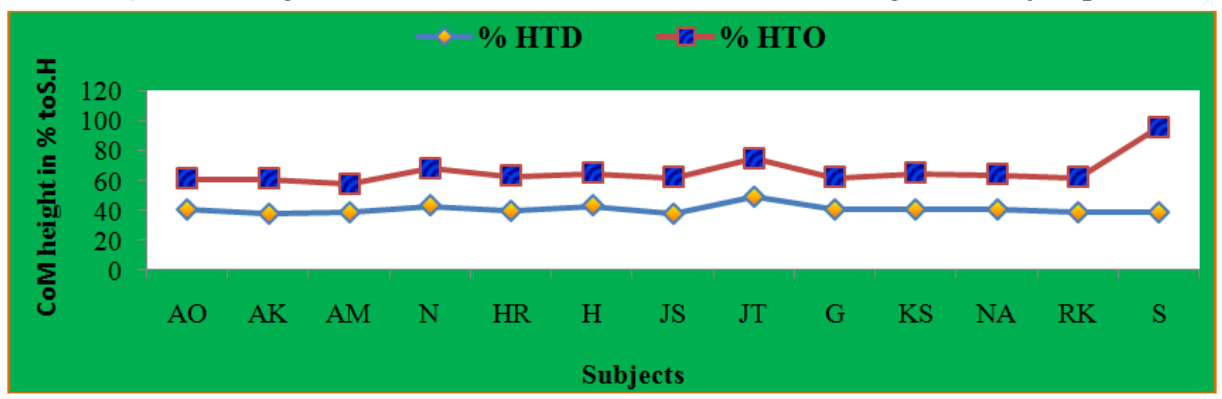

\section{Results and Conclusion}

In this competition majority floppers are speed jumpers. The majority of subjects centre of mass height at touchdown in relation to standing height (HTD\%) was lower than the elite athletes (previous studies of $49 \%$ reported by Dapena (1980a)Majority of subjects center of mass height at toe off in relation to standing height (HTO\%) was lower than that of the elite athletes. (71\% reported by Dapena(1980a).

According to Dapena (1980b) from back view (lateral trunk lean) the longitudinal axis of the trunk (i.e., the line going from the base of the neck to the midpoint between the hips) should be leaning about 15 degrees away from the bar at the start of the takeoff phase and it should not be tilting toward the bar more than 10 degrees beyond the vertical at the end of the takeoff phase. Majority of subjects' horizontal velocity ratio (from touchdown to toe off) was lower than that of elite athletesDapena et al. (1986) reported the average horizontal velocity was $7.37 \mathrm{mps}$ at touchdown and $3.92 \mathrm{mps}$ at takeoff $(53 \%)$. The majority of high jumpers vertical velocity at toe off was on par with elite high jumpers( Dapena (1980a) found that the 4.30mps at takeoff).Majority of subjects' take off time was similar to the elite athletes' (earlier studies of range of 0.140 to $0.200 \mathrm{sec}$ with mean of $0.160 \mathrm{sec}$ Dapena (1980a) The majority of subjects' knee angle at touchdown was lower than elite athletes (mean value of $168^{\circ}$ reported by JuhaIsolehto et al.) but majority of subjects' knee angle at toe off was on par with the elite athletes.

The researcher found that the height of center of mass, majority of subjects' at touchdown and at toe off in relation to their standing height was low. This study suggests that to increases the performance i.e. to clear a high jump bar, it is necessary to drive the center of mass of the athlete as high as possible by keeping COM heights at favorable position during takeoff phase.

These results also found that majority of high jumpers horizontal velocity at touchdown was good but horizontal velocity at toe off was high. The rate of decrease of horizontal velocity from touchdown to toe off was low when compared to the elite athletes. However, very few maintained the required rate of decrease. In case of vertical velocity at touchdown it was higher than the required but at toe off it was effective. This study suggests the athletes for conversion of horizontal velocity in to vertical velocity with minimal speed loss. As it 
requires greater elastic strength of the take-off leg muscles the athletes should develop elastic strength for optimum performance.

In case of movement timings majority of subjects' takeoff time was on par with elite athletes. The present results showed that majority of floppers' knee angle at touchdown was lower than elite athletes but knee angle at toe off was on par with the elite athletesFosbury flop high jump depends on anthropometric factors. Therefore being tall could be an advantage but a good position at takeoff could give optimum performance.

\section{Recommendations}

1. Since majority of the jumpers are unable to maintain the low center of mass at the touch down, it is recommended to incorporate more Plyometric exercises with weight so that they can maintain lower center of mass at the time of touchdown.

2. Since majority of jumpers are maintaining low horizontal velocity (from TD to TO) ratio they are unable to convert to vertical velocity. It is recommended to maintain optimum ratio i.e. 53\%. The trainers should regulate horizontal speed of jumper basing on the ability of the athlete to convert the horizontal velocity into vertical velocity.

3. Since majority of jumpers are unable to maintain the negative vertical velocity at touchdown it is recommended that high jumper may try to make the last two foot contacts with the ground very quickly one after the other. Further it is recommend to maintain the length of the last step is very long which could contribute to a late planting of the takeoff foot, and in turn lead to a large negative value at touchdown.

4. It is recommended to conduct further research on approach run through video analysis to understand clearly about its impact on performance (influencing factors on COM heights.)

\section{References}

[1]. Alexander, R.M. (1990). Optimum take-off techniques for high and long jumps.

[2]. Philosophical Transactions of The Royal Society, B329, 3-10.

[3]. Dapena, J. (1980a) Mechanics of $\mathrm{t} r$ a $n$ s 1 a t i o $\mathrm{n}$ i n the F0sbur.y-flop. e.d S ci . sports $\sim$ xercsie 12:37-44

[4]. Dapena, J. (1980b). Mechanics of rotation in the Fosbury-flop.Medicine and Science in Sports and fiercise, 12:45-53.-44

[5]. Dyatchkov, V.M. (1968). The high jump.Track Technique, 34:1059-1075.

[6]. JuhaIsolehto, MikkoVirmavirta, heikkikyrolanenandPaavo V. Komi(2007). Biomechanicalanalysis of the high jump at the 2005 IAAF World Championships in Athletics.IAAF New Studies in Athletics • no. 2/2007, 22:2; 17-27, 2007. 